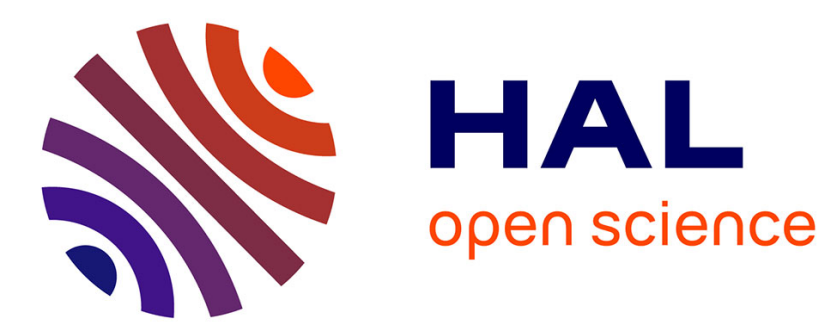

\title{
Le financement des collèges d'humanités en France à l'époque moderne (XVIe-XVIIIe siècle)
}

Boris Noguès

\section{To cite this version:}

Boris Noguès. Le financement des collèges d'humanités en France à l'époque moderne (XVIe-XVIIIe siècle). Le coût des études. Modalités, acteurs et implications sociales (XVIe-XXe siècle), PUR, pp.45-60, 2012, 10.4000/books.pur.113678 . halshs-00829198

\section{HAL Id: halshs-00829198 \\ https://shs.hal.science/halshs-00829198}

Submitted on 8 Jan 2019

HAL is a multi-disciplinary open access archive for the deposit and dissemination of scientific research documents, whether they are published or not. The documents may come from teaching and research institutions in France or abroad, or from public or private research centers.
L'archive ouverte pluridisciplinaire HAL, est destinée au dépôt et à la diffusion de documents scientifiques de niveau recherche, publiés ou non, émanant des établissements d'enseignement et de recherche français ou étrangers, des laboratoires publics ou privés. 


\section{Le financement des collèges d'humanités en France (XVI ${ }^{\mathrm{e}}-\mathrm{XVIII}{ }^{\mathrm{e}}$ siècles)}

\section{Boris Noguès}

Texte paru dans J.-F. Condette (éd.), Le coût des études. Modalités, acteurs et implications sociales (xvie-xxe siècle), p. 45-60, PUR, 2012

L'étude du financement des différentes institutions scolaires sous l'Ancien régime a pour l'heure donné lieu à un traitement très inégal. L'école élémentaire a indiscutablement été privilégiée, à travers plusieurs études régionales ${ }^{1}$. Même si aucune synthèse n'existe encore et si de nouveaux travaux seraient bien utiles, ces études couvrent des espaces et des situations suffisamment larges et variés pour fournir une image assez précise des pratiques en usage à ce niveau d'enseignement. En revanche, la question n'a guère été envisagée pour les autres types d'institutions éducatives de l'époque moderne, comme les séminaires, les collèges d'humanités, les écoles spécialisées dans la formation du personnel technique et militaire ou les académies nobles. Malgré leur caractère pionnier ou élitiste, qui justifierait à lui seul leur étude, les académies nobles et les écoles d'ingénieurs conservent un caractère marginal - ou pour le moins minoritaire - par rapport aux collèges d'humanités, tout comme les séminaires, qui relèvent d'un projet très particulier. Ceci invite donc à les laisser de côté, dans le cadre limité de cette étude, pour se concentrer sur la question du financement des collèges. Cette question n'a pour l'heure été abordée que dans le cadre des monographies d'établissement, sans qu'on n'ait jamais cherché à relier et à comparer les différents exemples étudiés, à l'exception des résultats provisoires et très synthétiques présentés par Marie-Madeleine Compère et Dominique Julia, à l'occasion de la rédaction des premiers tomes de leur répertoire des collèges français ${ }^{2}$. On dispose de ce fait d'une documentation surabondante mais complètement éclatée, dont on cherchera ici à donner un aperçu plus global. On ignore ainsi quelle est la participation effective des différents acteurs susceptibles de financer les collèges. Les historiens de l'école élémentaire ont pour leur part insisté, à l'intérieur de ce qu'on a pu identifier comme «la triade Église-État-communautés ${ }^{3}$ », sur le rôle tenu par les communautés locales dans le développement de cette institution, au tournant des XVII et $\mathrm{XVIII}^{\mathrm{e}}$ siècles, évoquant même à ce propos un "humanisme» villageois, réplique à une échelle plus modeste des pratiques urbaines envers le collège ${ }^{4}$. Mais le schéma ainsi dessiné soulève au moins trois types de questions. Il conviendrait avant tout de s'assurer que les municipalités ont effectivement tenu le premier rôle dans le financement des collèges. D'autre part, les différences entre les collèges et l'école élémentaire sont nombreuses et paraissent susceptibles d'invalider le parallélisme établi entre les deux institutions: le réseau des

\footnotetext{
${ }^{1}$ Dominique Julia, «L'enseignement primaire dans le diocèse de Reims à la fin de l'Ancien Régime », Annales historiques de la Révolution française, avril-juin 1970; Mireille Laget, « Petites écoles en Languedoc au XVIII ${ }^{\mathrm{e}}$ siècle », Annales. E.S.C., n 6, 1971, p. 1398-1418; Roger Chartier, Marie-Madeleine Compère et D. Julia, $L^{\prime}$ éducation en France du XVI au XVIII siècle, Paris, SEDES, 1976, p. 27-30 et p. 62-67 ; M.-M. Compère, «École et alphabétisation en Languedoc aux XVII et XVIII ${ }^{\mathrm{e}}$ siècles », in F. Furet et J. Ozouf, Lire et écrire. L'alphabétisation des Français de Calvin à Jules Ferry, Paris, Éditions de Minuit, 1977, t. II, p. 43-99; Dominique Blanc, "Les saisonniers de l'écriture. Régents des petites écoles en Languedoc au XVIII siècle », Annales, E.S.C., 1988, n4, pp. 867-895; Guy Astoul, Les chemins du savoir en Quercy et Rouergue à l'époque moderne. Alphabétisation et apprentissages culturels, Toulouse, Presses universitaires du Mirail, 1999.

${ }^{2}$ M.-M. Compère, D. Julia, «Les Collèges sous l'Ancien Régime. Présentation d'un instrument de travail», Histoire de l'éducation, 1981, $\mathrm{n}^{\circ} 13, \mathrm{p} .1-27$.

${ }^{3}$ D. Blanc, art. cit., p. 868.

${ }^{4}$ D. Blanc, art. cit., p. 867. Voir aussi M. Laget, art. cit., p. 1398.
} 
collèges se constitue au moins un siècle avant celui des petites écoles ; la part des héritages médiévaux est loin d'y être négligeable; les fonctions sont différentes (nature de l'enseignement, projet éducatif, public accueilli); le collège connaît lui-même des transformations importantes (triomphe du collège d'humanités congréganiste) ou subit celles qui touchent la France en général (confessionnalisation de la société, montée en puissance de l'État royal, réduction concomitante des libertés urbaines...). Enfin, la simple lecture de quelques comptes de collèges montre rapidement qu'il conviendrait d'ajouter à la «triade » identifiée par D. Blanc le patrimoine et les ressources propres aux établissements, ainsi que la participation des familles. La contribution de ces dernières reste dans tous les cas particulièrement difficile à déterminer, tant est floue la limite qui sépare l'enseignement proprement dit de l'éducation en général ou de l'entretien des enfants. Si les nécessités de l'exposition invitent à distinguer le financement de l'enseignement (salaires des professeurs, bâtiments scolaires) de l'entretien des élèves (prix de la pension, fournitures...), il paraît donc indispensable de prêter la plus grande attention aux liens qui existent entre les différentes dépenses ou les différentes ressources, en envisageant autant que possible le coût des études de manière globale. En effet, la porosité entre ces différents postes est certainement plus forte que ne le laissent supposer la documentation ou la logique institutionnelle. C'est dans cette perspective que seront présentées les différentes ressources des collèges, leur généalogie et leur renouvellement, avant d'esquisser une typologie des établissements fondée sur l'analyse du financement d'une trentaine d'établissements des XVII ${ }^{\mathrm{e}}$ et $\mathrm{XVIII}{ }^{\mathrm{e}}$ siècles.

\section{1-Les sources de financement traditionnelles}

\section{L'entretien du personnel enseignant}

Dans les universités médiévales, l'enseignement était semble-t-il gratuit à l'origine, pour des raisons théologiques: la science n'appartenant qu'à Dieu, les enseignants ne pouvaient en faire commerce ${ }^{5}$. La subsistance de ces hommes était donc assurée par des activités et des revenus qui n'avaient pas de lien direct avec l'enseignement. Les juristes et les médecins exerçaient une activité professionnelle parallèle et les régents des facultés de théologie et des arts étaient généralement titulaires de bénéfices ecclésiastiques ou appartenaient à un ordre religieux, ce qui revient à dire que leur enseignement était alors financé par l'Église. Cette subvention ecclésiastique se maintient tout au long de la période moderne, dans les facultés de théologie, mais aussi, même si elle régresse, dans bon nombre de collèges d'humanités. On en trouve encore des traces aux XVII ${ }^{\mathrm{e}}$ et $\mathrm{XVIII}^{\mathrm{e}}$ siècles, par exemple parmi ces professeurs des collèges de la faculté des arts de Paris titulaires de bénéfices (il est vrai de moins en moins nombreux et de plus en plus modestes, sous l'effet conjugué de la réforme tridentine et de l'amélioration de la condition enseignante dans cette ville $)^{6}$. De même, la dévolution dans les années 1770 et 1780 à des réguliers de collèges anciennement tenus par les jésuites, après l'échec de leur reprise par des séculiers ou des laïcs, renoue finalement avec les pratiques en vigueur dans les universités du XIII ${ }^{\mathrm{e}}$ siècle $^{7}$. Outre

\footnotetext{
${ }^{5}$ G. Post, K. Giocariniset et R. Kay, « The Medieval Heritage of Humanistic Ideal : Scientia Donum Dei Est, Unde Vendi Non Potest », Traditio, II, 1955, p. 196-234; Dante Zanetti « À l'Université de Pavie au XVe siècle : les salaires des professeurs », Annales. E.S.C., 1962, vol. 17, n 3, p. 421-433 ; J. Le Goff, Les intellectuels au Moyen Âge, Genève, 1965.

${ }^{6}$ Boris Noguès, Une archéologie de corps enseignant. Les professeurs des collèges parisiens aux XVII et XVIII siècles, Paris, Belin, 2006, p. 95-106.

${ }^{7}$ Plusieurs exemples de collèges confiés à des réguliers après l'échec des séculiers, à partir des années 1770 , Arch. nat., AD VIII 4.
} 
l'octroi de bénéfices ou l'intégration de réguliers, le financement de l'enseignement peut également reposer à l'époque moderne sur le monopole de la célébration de certains offices religieux par les enseignants de tel ou tel collège. C'est le cas dans le petit collège de Péronne, desservi depuis 1725 par les religieux Mathurins, où l'on nous apprend qu'ils ont l'exclusivité de la célébration de certaines messes et que, selon les administrateurs de la ville, le revenu du collège doit pour cette raison être augmenté de 600 livres $^{8}$. À Castelnaudary, en 1664, cette ressource paraît même assurer seule le fonctionnement de l'établissement qui n'est " à charge ni du roy ni au public, puisque le revenu provient de certains obits fondés par des particuliers ${ }^{9} . »$

Le financement de l'enseignement par l'Église ne s'explique pas seulement par la survie de pratiques archaïques, mais correspond largement aux conceptions des hommes de l'époque moderne, jusqu'à la fin de l'Ancien Régime. C'est en effet vers l'Église et ses riches bénéfices que l'on se tourne spontanément lorsqu'il s'agit de trouver ou compléter un financement en faveur d'une institution éducative, car l'enseignement, au même titre que la prédication, apparaît comme un des aspects de la fonction pastorale et, d'autre part, parce que la finalité affichée de toute éducation reste religieuse ${ }^{10}$. Parmi des dizaines d'autres exemples qui révèlent la place de ce mode de financement dans les conceptions du temps, en 1763, les oratoriens du collège de Niort font un bilan calamiteux des finances de leur collège et écrivent : "d'après ces considérations, les pères de l'Oratoire demandent qu' on [...] fasse la réunion à leur maison du prieuré de Niort, pour les mettre en état d'acquitter leurs dettes et de continuer de donner à la ville de Niort l'éducation des jeunes gens qu'elle renferme ${ }^{11}$. » C'est la solution qui est retenue pour renflouer le collège de Soissons, qui obtient la réunion des revenus d'un prieuré en 1773 et d'une abbaye en $1781^{12}$. Quelle que soit la région ou la période considérée, les bénéfices ecclésiastiques apparaissent bien, dans l'esprit des enseignants comme des administrateurs, comme la source à laquelle il convient naturellement de puiser.

L'illustration la plus systématique de cette conception du rôle de l'Église est fournie par l'ordonnance d'Orléans, qui décide en 1560 que dans chaque chapitre cathédral ou collégial de plus de dix chanoines, une prébende doit obligatoirement être réservée à l'enseignement. Cette prébende " préceptoriale » (à distinguer de la prébende "théologale », qui existe dans les cathédrales depuis le Moyen Âge pour l'enseignement de la théologie) constitue jusqu'à la fin de l'Ancien Régime une source très importante de financement des collèges, que l'on retrouve dans pratiquement tous les établissements (voir infra la situation des différents collèges) ${ }^{13}$. L'affectation obligatoire de cette prébende à l'enseignement et à un collège particulier ne va pas sans susciter dans un premier temps des résistances de la part de l'évêque et des chanoines, qui s'estiment lésés. En atteste l'arrêt du Parlement de Toulouse, du 12 février 1569, portant que l'abbé de Saint-Sernin, Baptiste de Simiane, évêque d'Apt, et le syndic du chapitre de Saint-Sernin payeront chacun par moitié au recteur du collège des jésuites de Toulouse la somme de 100 livres pour arrérages de la prébende préceptoriale qu'ils

\footnotetext{
${ }^{8}$ Comptes rendus aux chambres assemblées, par MM. les commissaires nommés par les arrêts des 6 août 1762 et 24 mars 1763 [de Laverdy, l'abbé Terray, Rolland, Roussel de La Tour], Paris, 1763 (recueil BnF 4-LD 39554), désormais abrégé « CRC », p. 102.

${ }^{9}$ François de Dainville, L'éducation des jésuites (XVIe-XVIIIe siècles), Paris, Éditions de Minuit, 1978, p. 139.

${ }^{10}$ R. Chartier et al., L'éducation en France..., op. cit., p. 26, cite les cahiers de doléances des États généraux de 1614 comme exemple de volonté de faire peser la charge sur l'Église. Voir aussi, M.-M. Compère, D. Julia, « Les collèges sous l'Ancien Régime », art. cit., p. 10-11.

${ }^{11}$ CRC, p. 99.

${ }^{12}$ CRC, p. 141 et M.-M. Compère et D. Julia, Les collèges français (XVI $-X V I I I^{e}$ siècles), Répertoire, t. 2, Paris, INRP-CNRS, 1988, p. 611.

${ }^{13}$ Voir Claude Joly, Traité historique des écoles épiscopales et ecclésiastiques..., Paris, 1679, p. 175.
} 
refusaient de verser ${ }^{14}$. Il convient surtout de souligner la transformation profonde qu'introduit ce mode de financement : en affectant le revenu des bénéfices à un établissement et non plus individuellement à un enseignant bénéficier, le système rompt le lien direct et personnel qui unissait les professeurs à l'Église. Désormais, l'appartenance de tous les enseignants au clergé ne correspond plus à une nécessité économique. Cette rupture dans la nature du corps enseignant s'opère de manière non linéaire - on observe une « sacerdotalisation » provisoire du corps au XVII ${ }^{\mathrm{e}}$ siècle, lorsque des prêtres congréganistes remplacent les régents séculiers et reste inachevée en 1789 , comme on l'a vu à Paris ${ }^{15}$. Mais elle s'inscrit globalement dans une double logique, celle de la réforme tridentine, du côté de l'Église, qui associe de plus en plus étroitement la détention d'un bénéfice ecclésiastique à une réelle activité pastorale et, d'autre part, celle d'une professionnalisation et d'une laïcisation des enseignants, qui devient nette chez les séculiers ou les oratoriens dans la seconde moitié du XVIII ${ }^{\mathrm{e}}$ siècle $^{16}$.

Avant même la fin du Moyen Âge, le financement ecclésiastique était de toute façon complété par d'autres, les préventions contre la rétribution du savoir évoquées plus haut s'étant rapidement effacées. Il est ainsi largement admis que les étudiants paient des droits pour obtenir des diplômes ${ }^{17}$. Comme les professeurs sont également les membres des jurys d'examen, ceci revient à les payer indirectement. La maîtrise es arts est cependant le seul diplôme qui concerne les collèges et tous les collèges ne peuvent compter sur cette ressource, car seulement une vingtaine de villes françaises disposent d'une faculté des arts active $^{18}$. De plus, tous les professeurs ne participent pas à la délivrance des grades. Pour ceux qui peuvent en bénéficier, on peut estimer que cette participation rapportait à Paris une centaine de livre à chacun vers $1765^{19}$. Dans le même registre, les collèges qui appartiennent aux facultés des arts peuvent éventuellement jouir des privilèges universitaires, comme des exemptions fiscales ou des ressources particulières. Le cas mérite surtout d'être mentionné à Paris, où le revenu des messageries universitaires est exclusivement affecté au salaire des enseignants à partir de 1634 et où un accord très avantageux est passé avec la monarchie en 1719. Cet accord permet d'augmenter considérablement les salaires des professeurs et de supprimer entièrement l'écolage payé par les familles, qui s'élevait alors à 36 livres par an dans la capitale ${ }^{20}$. Le financement de l'enseignement est donc, dans ce cas très particulier, assuré par les revenus tirés d'une ressource propre à l'université, sur laquelle la corporation enseignante a su mettre la main.

Mais cette gratuité est loin d'être la règle et dans bien des établissements les familles sont contraintes de payer pour l'enseignement de leurs enfants, comme d'ailleurs dans la plupart des écoles élémentaires rurales. Chaque mois ou chaque trimestre, les élèves apportent au maître leur écot. Au collège de Beauvais, au milieu du XVIII ${ }^{\mathrm{e}}$ siècle, on apprend ainsi que "les écoliers paient [...chaque année] en philosophie 12 livres; en rhétorique 10 livres 10

\footnotetext{
${ }^{14}$ Inventaire des Arch. Municipales de Toulouse, AA20/122.

${ }^{15}$ B. Noguès, op. cit., p. 95-106.

${ }^{16}$ Willem Frijhoff et Dominique Julia, «Les oratoriens de France sous l'Ancien Régime. Premiers résultats d'une enquête », Revue d'histoire de l'Église de France, 1979, p. 227-265 et, des mêmes, « Le recrutement d'une congrégation enseignante et ses mutations à l'époque moderne: l'Oratoire de France. », Historical Reflections, vol 7, Nos 2\&3, 1980, p. 443-458 ; B. Noguès, op. cit., p. 187-190.

${ }^{17}$ Voir Ramus (Pierre de la Ramée), Advertissement sur la réformation de l'université de Paris, au Roy, Paris, impr. A. Wechel, 1562.

18 Abbé Pelletier, «La réitération de grades », Bulletin de la société archéologique de l'Orléanais, $\mathrm{n}^{\circ} 33$, $2^{\mathrm{e}}$ trimestre 1859 , p. 114.

${ }^{19}$ B. Noguès, « La maîtrise ès arts en France aux XVII ${ }^{\mathrm{e}}$ et XVIII ${ }^{\mathrm{e}}$ siècles. Rites universitaires, épreuves scolaires et usages sociaux d'un grade », Histoire de l'éducation, n 124, 2009, p. 95-134, p. 104.

${ }^{20}$ «Calcul de ce que les professeurs des anciens collèges reçoivent de leur messageries...», cité par Ch. Jourdain, Histoire de l'université de Paris aux XVII et XVIII siècles, pièces justificatives ${ }^{\circ}{ }^{\circ}$ CXLVI, p. 126. Voir aussi B.N.F., Z-Thoisy-322, fol. 150.
} 
sols [...] ; et ainsi en diminuant jusqu'en sixième, où on paie 4 livres 10 sols $^{21}$. " Mais le versement de frais de scolarité par les élèves est considéré comme un pis aller qu'il serait préférable d'éviter, comme l'expliquent les officiers municipaux de Soissons en 1763, qui déclarent souhaiter "supprimer la contribution sur les étudiants, qui malgré le soin que l'on a d'en exempter les plus mal-aisés, est encore pénible aux autres et blesse la délicatesse des prêtres de l'Oratoire ${ }^{22}$. " Sont ici exempts d'écolage les pauvres reconnus, c'est-à-dire ceux qui ont accepté d'entamer une démarche auprès des autorités municipales et de leur curé, qui peut leur rédiger un billet attestant de leur indigence. Dans quelques villes, comme à Annecy, tous les enfants de la cité ou des faubourgs sont dispensés d'écolage, qui repose sur les élèves forains $^{23}$.

\section{Les dépenses à la charge des familles}

Mais, même si ces frais d'écolage paraissent lourds aux familles et aux administrateurs, ils ne représentent qu'une faible part de l'ensemble de la dépense éducative, qui est pour l'essentiel occupée par l'entretien de l'élève. En effet, le coût de la pension - qui fournit un ordre de grandeur des sommes nécessaires à cet entretien - s'élève en général au $\mathrm{XVIII}^{\mathrm{e}}$ siècle à 200 ou 300 livres, souvent davantage, alors que, comme on l'a vu avec les exemples précédents, l'écolage ne représente que 5 à $10 \%$ de cette somme ${ }^{24}$. Pour les familles, le coût total d'une scolarité est, en plus de la pension ou de l'entretien familial, augmenté des dépenses "accessoires », qui s'élèvent avec le niveau social de la famille: coûts d'habillement, de toilette; achat des livres de classe; exercices ou soutenances publiques les plus aisés, qui peuvent se monter à plusieurs centaines de livres ${ }^{25}$. Surtout, toute une série de leçons particulières consacrées aux arts d'agrément, comme la musique, la danse, ou l'escrime constituent le complément indispensable de l'enseignement classique pour les élites. Au XVIII ${ }^{\mathrm{e}}$ siècle, les leçons d'un maître à danser peuvent coûter 3 livres par mois, soit bien plus que la rémunération due l'enseignement dans la classe de rhétorique ${ }^{26}$. Au final, les coûts globaux à la charge des familles dépassent parfois 2000 livres par an et par enfant au $\mathrm{XVIII}^{\mathrm{e}}$ siècle $^{27}$. Le pensionnat oratorien de Juilly, au recrutement social sélectif il est vrai, en donne une bonne idée lorsqu'il propose en 1786 un "abonnement» forfaitaire, tout frais inclus, avec la pension, l'habillement, l'infirmerie, les livres et les cours d'agrément, pour 900 livres $^{28}$. À l'autre extrémité de l'échelle sociale, existe cependant la solution inverse, destinée à minimiser le coût pour les familles en réduisant les services offerts : la pension sans la nourriture, comme au collège de Beauvais, où l'on explique en 1763 qu'il «y a dans ce collège dix ou douze pensionnaires qui paient 250 livres par an, et environ soixante enfants de laboureurs et gens de campagne qui sont logés moyennant 32 livres et qui se nourrissent eux-mêmes ", les enfants rapportant certainement de la ferme familiale des denrées nécessaires ${ }^{29}$.

\footnotetext{
${ }^{21}$ CRC, p. 7.

${ }^{22}$ CRC, p. 141

${ }^{23}$ Serge Tomamichel, Le Collège D'Annecy au XVI Siècle. Une école de la Réforme Catholique ?, Paris, 1999, p. 65 .

${ }^{24}$ L'ordre de grandeur pour les pensions est tiré de la lecture de M.-M. Compère et D. Julia, Les collèges français..., op. cit. Voir aussi F. de Dainville, op. cit., p. 138, qui calcule qu'en 1688, pour 3658 élèves, le financement des collèges (strictement limité à l'activité d'enseignement des humanités) ne revient qu'à 31000 livres, soit 8 livres par an et par élève.

${ }^{25}$ F. de Dainville, op. cit., p. 289-290 pour les livres de classe ; B. Noguès, art. cit., p. 107-110 sur le coût des soutenances publiques.

${ }^{26}$ F. de Dainville, op. cit., p. 290.

${ }^{27}$ R. Chartier, et al., L'éducation en France..., op. cit., p. 180.

${ }^{28}$ M.-M. Compère et D. Julia, Les collèges français..., t. 2, op. cit., p. 342.

${ }^{29}$ CRC, p. 7
} 
Il existe bien depuis le Moyen Âge des centaines de bourses, fondées par des particuliers, qui ont justement vocation à assurer l'entretien gratuit des étudiants et soulager d'autant les familles. Mais, outre la mainmise des notables sur la collation de ces bourses, qui en réservent l'usage à leurs parents et alliés, on assiste au cours de la période moderne à une véritable crise du système des bourses, qui souffrent d'une dévaluation générale de leurs revenus ${ }^{30}$. Philippe Marchand l'a relevé à propos des bourses du collège de Lille entre 1692 et $1712^{31}$. On le constate au fil des enquêtes qui examinent la situation financière des collèges parisiens aux $\mathrm{XVII}^{\mathrm{e}}$ et $\mathrm{XVIII}^{\mathrm{e}}$ siècles. En 1642, le recteur constate ainsi que, faute de ressources, le collège de Reims n'accueille plus aucun boursier, contrairement aux statuts de sa fondation, et qu'il s'est transformé en établissement fantôme, tenu par un principal et un procureur dont on se demande ce qu'ils y font ${ }^{32}$. Le bilan dressé en 1762 dans la capitale montre finalement que la moitié des bourses fondées y est en déshérence, car comme l'explique L'Averdy "Les fondateurs ne doutant pas que les biens des collèges ne fussent tous conduits avec une prudente économie, avaient prévu comme certain le cas de l'augmentation de ces biens; tandis qu'au contraire ils sont diminués considérablement dans beaucoup de collèges, anéantis dans d'autres, les bourses supprimées ou réduites ${ }^{33}$. " La situation est comparable en province : à la même date, le constat est le suivant à Troyes : «ces fondation et autres [...] devraient former un principal de 30000 livres, mais une partie s'est trouvée absorbée par les révolutions qu'ont éprouvé les rentes, et une autre partie a été employé à suppléer l'insuffisance du revenu pour la dépense courante ${ }^{34}$. "

Outre la dégradation des revenus des bourses déjà existantes, on observe à l'époque moderne un tarissement du mouvement de fondation des bourses. L'historique des bourses parisiennes réunies à Louis-le-Grand, dressé en 1763, montre ainsi que $84 \%$ des bourses ont été fondées avant $1500,7 \%$ au $\mathrm{XVI}^{\mathrm{e}}$ siècle, $4 \%$ au XVI ${ }^{\mathrm{e}}$ et $5 \%$ entre 1700 et $1763^{35}$. Il semble qu'à l'époque moderne les fondations charitables se réorientent plus volontiers vers d'autres objets que l'entretien des élèves. Elles privilégient désormais le financement direct de l'enseignement, à travers la création des écoles de charité ou la subvention d'un collège ou d'une chaire. Parmi tant d'autres, l'histoire des fondations en faveur du collège de Narbonne est exemplaire de ce mouvement : en 1530 y sont fondées quatre bourses (les dernières de la période moderne), en 1623 une chaire de philosophie, en 1644 deux chaires de théologie ${ }^{36}$. Typique également, cette fondation évoquée dans le mémoire consacré au collège de Beauvais : " le 18 août 1626, Pierre de Paris a laissé tout son bien au collège [...] pour y fonder une chaire de philosophie, à la charge de faire dire tous les ans une prière à la Sainte Vierge. Ce legs a monté à environ 6174 livres $^{37}$ ». Cent ans plus tôt, ses prédécesseurs

\footnotetext{
${ }^{30}$ Voir par exemple Arch. nat. AD VIII 4 A : Etat des bourses des collèges réunis à celui de Louis-le-Grand, 1781, «Troisième état. Bourses affectées à certaines familles »; Sur le caractère familial des bourses et leur décrépitude financière voir aussi L'Averdy, Compte-rendu aux Chambres, par M. L'Averdy, concernant la réunion des boursiers fondés dans les collège de non-plein exercice sis en la ville de Paris, 12 novembre 1763, sl, 1763, p. 16-71.

${ }^{31}$ Philippe Marchand, "Bourses et boursiers du collège de Lille sous l'Ancien Régime », Liber amicorum Claude Lannette, Lille, Commission historique de Nord, 2001, p. 251-261, p. 254 sur la baisse des revenus ; p. 258 sur la réservation des bourses par les familles riches.

${ }^{32}$ C. Jourdain, Histoire de l'université de Paris, édition de 1866, t. 1, p. 266.

${ }^{33}$ L'Averdy, Compte-rendu...concernant la réunion des boursiers, op. cit., p. 76 pour le bilan comptable, p. 6 pour la citation.

${ }^{34}$ CRC, p. 244.

35 Statistique dressée à partir de l'historique des fondations de bourses dans les collèges parisiens donnée par L'Averdy, Compte-rendu...concernant la réunion des boursiers, op. cit., p. 16-71. N'ont été retenues que les bourses destinées à des étudiants (artiens ou théologiens), à l'exclusion de celles destinées aux officiers (chapelains, principaux...), et fondées dans les collèges de la faculté des arts réunis à Louis-le-Grand.

${ }^{36}$ M.-M. Compère et D. Julia, Les collèges français ..., t. 1, op. cit., p. 483-485.

${ }^{37}$ CRC, p. 6-7.
} 
auraient sans doute voué cette somme «à l'entretien perpétuel » de quelques «pauvres » boursiers plutôt qu'au financement direct de l'enseignement.

\section{2-Nouveaux acteurs et nouvelles structures de l'économie de l'éducation aux XVII ${ }^{\mathrm{e}}$ et XVIII ${ }^{\mathrm{e}}$ siècles}

La réorientation des fondations en faveur de l'enseignement et au détriment du système des bourses provoque indiscutablement un transfert de certaines charges vers les familles. Cette réorientation s'explique, au moins en partie, par la mise en place des collèges d'humanités, qui commence à la fin $\mathrm{du} \mathrm{XVI}^{\mathrm{e}}$ siècle et se poursuit tout au long du siècle suivant $^{38}$. Ces collèges proposent en effet un encadrement pédagogique et disciplinaire des élèves beaucoup étroit que celui des facultés des arts. Ils disposent pour ce faire de bâtiments propres et d'un personnel d'enseignement et d'encadrement plus nombreux et plus spécialisé que ne l'étaient les bacheliers médiévaux, ce qui les conduit à supporter des frais fixes importants. Comme on vient de le voir, des fondations privées (ou parfois des donations de princes ou de dignitaires ecclésiastiques) contribuent tout au long de la période à ces charges. Les jésuites, qui jouent un rôle majeur dans la constitution de ce nouveau réseau d'établissements, sont les premiers à bénéficier de ces largesses ${ }^{39}$. Mais il convient d'insister sur l'apparition à la période moderne de nouveaux acteurs, qui étaient absents ou avaient une place très secondaire dans le fonctionnement des universités médiévales et dont le rôle reste à préciser : les villes, l'État et les congrégations enseignantes.

\section{Villes et État}

L'analyse des comptes de quelques dizaines de collèges montre que l'effort financier consenti par les villes est souvent - mais pas toujours - important ${ }^{40}$. Cet effort paraît même dans certains cas démesuré : en 1762, lors de l'expulsion des jésuites et la fermeture du collège, la ville de Hesdin réclame au Trésor la restitution des frais engagés en faveur de cet établissement depuis 1613, qu'elle estime à la somme totale de 88588 livres $^{41}$. Au-delà de ces exemples spectaculaires, et avant d'essayer d'évaluer le poids et la fréquence de l'investissement urbain en France (voir infra), on peut présenter les modalités de cette association entre la ville et le collège local. Le contrat signé le 14 septembre 1567 entre la ville de Lyon et la Compagnie de Jésus, lors de l'ouverture du collège de La Trinité, fournit une bonne illustration des relations qui unissent les deux parties. Ce contrat stipule que les bâtiments existants du collège et leurs dépendances sont donnés à perpétuité par la ville à la Compagnie, que les échevins fourniront " une pension et une provision annuelle de 800 livres tournois" et qu'en "outre demeureront au profit dudit collège tous fruits et revenus temporels et legs qui auront été donnés par le corps des habitants de ladite ville [de Lyon]. » En échange, de ces trois éléments, les jésuites s'engagent à "établir et entretenir en icelui collège un recteur et principal accompagné de personnes doctes et idoines de leur profession, en nombre suffisant pour régenter la jeunesse en tous arts libéraux [...et] endoctriner en

\footnotetext{
${ }^{38}$ M.-M. Compère, D. Julia, « Les collèges sous l'Ancien Régime », art. cit., donne une aperçu général de la constitution de ce réseau.

${ }^{39}$ Voir Henri Fouqueray (le P.), Histoire de la Compagnie de Jésus en France, des origines à la suppression (1528-1762), 4 tomes, en particulier les t. 1 et 2, qui multiplie les récits détaillés des fondations de collèges, et donne, malgré sa perspective apologétique, une bonne idée des forces qui favorisent la venue des jésuites.

${ }^{40}$ Le rôle des villes dans la fondation des collèges a été souligné par F. de Dainville, op. cit., p. 153, M.-M. Compère, D. Julia, « Les collèges sous l'Ancien Régime », art. cit., p. 7-9 et R. Chartier et al., op. cit., p. 166.

${ }^{41}$ CRC, p. 304-305.
} 
toute piété et en la foi catholique tous les dits écoliers [...] le tout gratuitement selon les saintes doctrines et ordonnances ${ }^{42}$. " Une autre illustration de l'interventionnisme municipal est fournie par la politique menée à Provins, à la fin du XVII ${ }^{\mathrm{e}}$ siècle : "Ce collège, dit le supérieur, a été desservi durant plusieurs années par des séculiers; mais étant demeuré vacant pendant environ trois ans, les maire et échevins en 1669 appelèrent les soi-disant jésuites, avec lesquels ils traitèrent aux conditions de les faire jouir $1^{\circ}$ des revenus de la maladrerie de Close-Barbe et dépendances $2^{\circ}$ Des deux préceptoriales [...] $3^{\circ} \mathrm{Du}$ droit de la courte Pinte [taxe municipale sur les boissons] ${ }^{43}$ " Plusieurs traits généraux méritent d'être relevés à partir de ces deux exemples. Comme pour le maître d'école embauché par la communauté rurale, on note le caractère contractuel des liens entre la ville et l'établissement scolaire, qui était absent dans la fondation des universités ${ }^{44}$. D'autre part, les villes fournissent pratiquement toujours deux choses au collège local: des bâtiments, avec le mobilier nécessaire, destinés à l'exercice, qu'ils s'agisse d'anciens bâtiments conventuels reconvertis ou de nouveaux et, un peu moins systématiquement, un revenu destiné à entretenir les enseignants. Ce versement prend la forme à Provins de l'abandon des revenus de biens appartenant à la ville et du produit d'une taxe, mais peut ailleurs consister en une pension, comme à Toulouse, où on peut citer un arrêt du Parlement "enjoignant aux capitouls de payer au syndic du collège des Jésuites une pension annuelle de 1200 livres pour la nourriture et entretien des régents ${ }^{45}$. " On retrouve par ailleurs à Lyon mention des futures fondations privées destinées à grossir les revenus du collège et à Provins une allusion aux prébendes préceptoriales. Il convient de souligner que derrière les "villes » interviennent en réalité les notables urbains, qui jouent un rôle moteur dans ce mouvement et utilisent les ressources locales pour offrir sur place une éducation à leurs enfants ${ }^{46}$. Les officiers de la ville de Provins justifient ainsi la présence dans leur ville d'un collège oratorien, estimant que "supprimer le collège de Provins, ce serait priver les jeunes gens de la ville et ceux des environs d'une pension que leurs parents ne pourraient leur procurer ailleurs, et en même temps les empêcheraient de pouvoir aspirer aux charges de leurs pères ${ }^{47}$." Même une minuscule bourgade comme Crespy-en-Valois n'hésite pas, au milieu du XVIII ${ }^{\mathrm{e}}$ siècle, à exiger le maintien de son collège, en affirmant que " la ville est considérable et renferme plus de 2000 habitants, deux villages lui servent de faubourgs ${ }^{48}$. "

Mais si les « villes » jouent un rôle de premier plan dans la création et le financement d'un établissement, d'autres autorités, traditionnelles ou nouvelles, peuvent également intervenir à cette occasion. Ainsi à Boulogne-sur-Mer "il a été proposé en 1628 à la congrégation de l'Oratoire de se charger de ce collège et l'affaire a été terminée par un acte du 6 juillet 1629 entre la Congrégation d'une part, l'Evêque, le chapitre, le baillage et les Mayeur, Echevins et élus de la communauté de l'autre part ${ }^{49}$. "Figurent ici dans le contrat, en plus de la ville, qui fournit le bâtiment et 300 livres, l'évêque, le chapitre et le baillage. L'évêque intervient à double titre, comme pasteur de la réforme tridentine intéressé à l'éducation, et comme contributeur, puisqu'il consent à abandonner une petite partie de ses revenus au collège. Le chapitre est présent à Boulogne en tant que contributeur, légalement obligé depuis l'ordonnance d'Orléans de 1560 d'abandonner une prébende au collège. Quant

\footnotetext{
${ }^{42}$ Cité par H. Fourqueroy, op. cit., t. 1, p. 400.

${ }^{43}$ CRC, p. 102.

${ }^{44}$ D. Blanc, art. cit., p. 868 et p. $874-875$.

${ }^{45}$ Inventaire des Arch. Municipales de Toulouse, AA18/329, arrêt du 29 janvier 1574.

${ }^{46}$ F. de Dainville, op. cit., p. 141 ; M.-M. Compère, D. Julia, « Les collèges sous l'Ancien Régime », art. cit., p. 9.

${ }^{47}$ CRC, p. 105.

${ }^{48}$ CRC, p. 52

${ }^{49}$ CRC, p. 9.
} 
au bailli, il manifeste sans doute en tant qu'officier royal la présence de l'État, mais sa contribution financière est nulle dans ce cas.

D'une manière générale, la participation de l'État au financement des collèges reste limitée à un seul mode d'action : imposer ou soutenir l'orientation de ressources locales vers l'éducation, comme pour l'école élémentaire ${ }^{50}$. Il utilise pour ce faire une loi de portée générale, l'ordonnance d'Orléans, et fournit un appui ponctuel aux communautés urbaines qui sollicitent l'autorisation de lever de nouveaux impôts locaux pour financer un collège ${ }^{51}$. Henri IV encourage ainsi la fondation du collège de Béziers en 1598: "Ce monarque ordonna qu'il fut levé deux sols par minots de sel qui se débiterai aux greniers de Narbonne, Peyriac, Sijean, Béziers, Pezenas, Marseillan, Mèze et Frontignan, jusqu'à la somme de 3000 écus [soleil], pour être employés à la construction du collège de Béziers [...] qui retire une pension de la crue du sel [.....$^{52}$ » L'exemple a le mérite de dessiner l'aire géographique qui contribue fiscalement à l'entretien de cet établissement, même si la justification de cet impôt et la clé de répartition restent obscures (Peyriac, Sijean, Marseillan, Mèze et Frontignan n'ont pas de collège et profiteront peut-être du nouvel établissement en y envoyant leurs enfants ; Pézenas est sur le même pied que Béziers, car un collège vient d'y ouvrir et profite également de cette ressource ; Narbonne est déjà doté d'un collège et ne semble tirer aucun bénéfice de cette nouvelle contribution ${ }^{53}$ ). Le roi fait aussi parfois un don sur le Trésor à quelque collège particulier, comme c'est le cas à La Flèche, somptueusement doté par Henri IV, ou à Louis-leGrand, placé sous le patronage de Louis XIV, qui lui aurait donné plus de 53000 livres en $1682^{54}$. Mais si les sommes engagées sont très importantes, il ne s'agit là que d'actions ponctuelles, qui ne se différencient pas par nature des fondations privées. Elles ne constituent donc pas une politique éducative de l'État, qui se contente dans ce domaine d'encadrer et de soutenir les initiatives locales.

\section{Congrégations et pensionnats : de nouveaux cadres pour l'éducation}

L'implantation des congrégations enseignantes, qui donne sa pleine mesure dans la première moitié du XVII ${ }^{\mathrm{e}}$ siècle, n'est pas sans conséquences sur le modèle économique des institutions d'éducation. Ces congrégations, qui fonctionnent à une échelle nouvelle, plus large que l'établissement (même si c'est dans ce cadre qu'elles déploient leur activité), apparaissent parfois, aux yeux des administrateurs, comme un moyen commode de réduire les coûts de fonctionnement du collège d'humanités tout en s'assurant le concours d'un personnel enseignant stable et fiable. C'est le cas à Annecy, où le déficit chronique du collège séculier

\footnotetext{
50 D. Blanc, art. cit., p. 885 : «L'intendant refuse systématiquement d'intervenir sauf pour autoriser une imposition scolaire ou sanctionner le trouble de l'ordre public. [...] L'administration provinciale ne se désintéresse pas du problème de l'école, du moins pas toujours, mais elle entend exercer une tutelle et non administrer directement en ce domaine. » Voir aussi M. Laget, art. cit., p. 1398-99.

${ }^{51}$ M.-M. Compère, D. Julia, « Les collèges sous l'Ancien Régime », art. cit., p. 12-13 donne d'autres exemples d'autorisations de lever des impôts tout au long du XVII ${ }^{\mathrm{e}}$ siècle.

${ }^{52}$ Arch. nat., F17/1314/A, pièce 4-1.

${ }^{53}$ M.-M. Compère et D. Julia, Les collèges français..., t. 1, op. cit., pour Narbonne p. 483 sq ; pour Pézenas p. 532.

${ }_{54}$ Arch. nat., M. 247, État des revenus et des charges du collège royal des jésuites de la Flèche en Anjou au mois d'octobre 1761, cité par Camille de Rochemonteix, Un collège de jésuites aux XVII et XVIII siècles. Le collège Henri IV de La Flèche, Le Mans, 1889, t. 1, p. 241-242 : " Les biens de fondation, outre l'emplacement et les bâtiments du collège, ses jardins et son parc, sont l'abbaye de N. D. de Bellebranche, et le prieuré-cure de Luché au diocèse du Mans, l'abbaye de Mélinais et les prieurés de Saint-Jacques et de Lechesnau au diocèse d'Angers, et un droit sur les papegaux de l'arc et de l'arbaleste de la province de Bretagne. » Le revenu net de ces fondations royales est estimé par Rochemonteix, une fois déduites les charges, à 19000 livres annuelles (p. 252). Pour Louis-le-Grand, CRC, p. 40.
} 
conduit en 1614 la ville à appeler les Barnabites ${ }^{55}$. Dans le même registre, la modestie des émoluments dont se contentent au milieu du XVIII ${ }^{\mathrm{e}}$ siècle les Minimes à Calais (seulement 250 livres annuelles par professeur, à peine mieux qu'un journalier) en fait certainement une excellente affaire pour cette localité ${ }^{56}$. En concurrence avec les séculiers, qu'ils éliminent dans bien des places aux $\mathrm{XVI}^{\mathrm{e}}$ et $\mathrm{XVII}^{\mathrm{e}}$ siècles, les congréganistes présentent en effet l'avantage de ne pas être obligés de verser de salaire à leurs membres. Ils ont également la possibilité d'opérer des transferts financiers à l'échelle de l'ordre entier, lorsque le besoin s'en fait sentir. Le cas est connu pour les jésuites, mais on en trouve également mention chez les oratoriens, où on note à propos du collège de Soissons en 1763 "que de tous temps ce collège a été hors d'état de se soutenir par lui-même et ne s'est réellement soutenu qu'en mettant à contribution les autres maisons de cette congrégation ${ }^{57}$. "

Cependant, si ces congrégations offrent, par leur taille et leur organisation, des garanties de bon fonctionnement supérieures à celles que pourrait proposer un principal prenant seul un établissement à bail, certaines font payer cher cette solidité. L'argument économique ne joue donc pas toujours un rôle de premier plan dans le choix d'appeler les jésuites ou les oratoriens. Les jésuites, qui ont refusé de s'installer à Annecy en 1614 comme ils y étaient invités, sont ainsi particulièrement exigeants et prudents lorsqu'ils acceptent de prendre en charge un collège, dans le droit fil des consignes de données dès 1593 par leur général $^{58}$. Il est vrai qu'ils peuvent se le permettre, car ils entament leur implantation dès la fin du XVI ${ }^{\mathrm{e}}$ siècle, période où ils sont sans concurrents, où la demande des villes est forte, où les régents séculiers ont souvent failli et où ils sont portés par le mouvement de reconquête catholique $^{59}$. De plus, ils bénéficient souvent du soutien de l'évêque local, prêt à concéder des unions de bénéfices, et jouissent par la suite de dons de particuliers très importants, ce qui explique leur prospérité dans la seconde moitié du XVII ${ }^{\mathrm{e}}$ siècle. Profitant de ce rapport de forces favorable, ils parviennent donc à choisir les meilleures places (une majorité est située dans les villes de plus de 10000 habitants $^{60}$ ), à des conditions très avantageuses. À Moulins par exemple, en 1604, la ville s'engage à fournir une maison de campagne pour les récréations et les vacances, en plus de tout ce qu'on trouve habituellement ${ }^{61}$. Ces implantations nouvelles sont donc également l'occasion de remobiliser des soutiens au profit d'une formule neuve et dynamique et, finalement, d'attirer vers le collège des ressources plus abondantes. Contrepartie de ces exigences élevées, l'enseignement est entièrement gratuit pour les externes dans les collèges jésuites, gage d'un succès immédiat et d'une popularité certaine. Fondés plus tard, les oratoriens, ne parviennent souvent à se faire une place qu'au prix d'exigences financières moins élevées, ce qui les contraint à imposer des droits d'écolage.

Après la constitution de ce réseau de collèges, réalisée pour l'essentiel au XVII ${ }^{\mathrm{e}}$ siècle, l'équilibre économique des établissements est affecté par une autre transformation des institutions d'éducation: l'association de plus en plus systématique d'un pensionnat au collège d'exercice (évolution entamée à la fin du XVII ${ }^{\mathrm{e}}$ siècle et restée inachevée, puisque la majorité des collèges n'a toujours pas de pensionnat à la fin du siècle XVIII ${ }^{\mathrm{e}}$ siècle $^{62}$ ). En effet, malgré la distinction comptable des activités d'enseignement et d'hébergement, le pensionnat, dont on a vu plus haut qu'il brassait des sommes plus importantes que l'exercice, est souvent utilisé comme une source de financement indirect du collège. Au collège de

\footnotetext{
${ }^{55}$ S. Tomamichel, op. cit., p. 153 et p. 190-191.

${ }^{56}$ CRC, p. 27.

${ }^{57}$ CRC, p. 141.

${ }^{58}$ R. Chartier et al., op. cit., p. 167.

${ }^{59}$ Les étapes de l'expansion jésuite ont maintes fois été décrites, par H. Fouqueroy, op. cit., F. de Dainville, op. cit., p. 58 sq et R. Chartier et al., op. cit., p. 167 et 186-190.

${ }^{60}$ R. Chartier et al., op. cit., p. 167 et p. 188.

${ }^{61}$ H. Fouqueray, op. cit., t. 3, p. 100.

${ }^{62}$ M.-M. Compère, D. Julia, « Les collèges sous l'Ancien Régime », art. cit., p. 16 et p. 19.
} 
Condom, en 1679, un inspecteur estime que sur une pension de 200 livres, le pensionnat fait 61 livres de bénéfices, au profit du collège ${ }^{63}$. À Lodève, en avril 1792, on déplore que, suite aux événements politiques, "la pension établie dans le collège et dont les profits le faisaient aller rondement étant tombée [...], on a perdu par là les moyens de suppléer à la modicité des fonds [du collège d'exercice $]^{64}$. " C'est également le bilan qui est tiré à la même date dans un mémoire sur le collège de Nantua, tenu par les prêtres missionnaires de la congrégation de Lyon, qui ne trouve son équilibre économique que grâce à la pension ${ }^{65}$. À l'inverse, cette enquête rétrospective réalisée en 1791 et 1792 montre que beaucoup d'établissements d'exercice sans pensionnat connaissent des difficultés financières, malgré l'empilement de ressources qu'on a présenté plus haut ${ }^{66}$.

Le premier moyen de faire payer l'enseignement aux pensionnaires consiste à faire porter sur les comptes du pensionnat la nourriture des professeurs ou les charges d'entretien des bâtiments, y compris les salles de classes. On nous l'apprend par exemple à Chinon, au milieu du XVIII ${ }^{\mathrm{e}}$ siècle : "des sommes ont été empruntées pour rétablir les anciens bâtiments $d u$ collège et ne sont pas à charge, attendu que le principal en paye une partie [grâce aux revenus $d u$ pensionnat $]^{67} »$. Autre moyen de financement indirect, la confusion du personnel des deux structures, avec un principal qui exploite traditionnellement le pensionnat, se paie sur ce dernier, mais fournit un encadrement gratuit au collège d'exercice. On peut également citer ces préfets de chambre jésuites qui sont en même temps professeurs du côté du collège d'exercice, ce qui rejoint l'usage qu'avaient traditionnellement les enseignants de prendre à titre privé quelques pensionnaires dans leur logement, contre rémunération ${ }^{68}$. Le pensionnat paraît d'une manière générale beaucoup plus rentable que le collège d'exercice ${ }^{69}$. On voit donc se développer au XVIII ${ }^{\mathrm{e}}$ siècle un nouveau modèle de collège, dont le financement repose largement sinon exclusivement sur le pensionnat. C'est le cas des grandes pensions oratoriennes de Juilly ou de Condom, au recrutement socialement très sélectif ou, pour prendre un exemple plus modeste, du pensionnat de Thirion, dans le baillage de Chartres dont on nous présente en 1763 l'histoire édifiante : "Les Bénédictins de la congrégation de Saint Maur étant entrés dans l'abbaye de Thirion, y établirent un petit collège et y enseignèrent gratuitement les humanités à ceux qui se présentèrent. [...] Le collège étant devenu florissant, le nombre de pensionnaires [ayant] augmenté, les religieux de Thirion firent construire d'autres bâtiments et employèrent, suivant eux, plus de 150000 livres pour mettre ce collège en règle et lui procurer toutes les commodités nécessaires. Cet établissement n'a aucune fondation et il n'y a aucune réunion de bénéfices. C'est l'abbaye qui fournit aux besoins, quand les pensions ne suffisent pas pour son entretien ${ }^{70} . "$

\section{3- Une typologie des établissements à travers l'origine de leurs recettes}

Ce tour d'horizon des différentes ressources possibles achevé, reste évaluer le poids de chacune d'elles dans le fonctionnement des collèges. Cette évaluation pose un certain nombre

\footnotetext{
${ }^{63}$ M.-M. Compère, D. Julia, Les collèges français..., op. cit., t. 1, p. 250.

${ }^{64}$ Arch. nat., F17/1314/A, pièce 3.

${ }^{65}$ Arch. nat., F/17/1311, pièce 10.

${ }^{66}$ Enquête révolutionnaire de 1790-1792 sur les établissements d'instruction publique (Arch. nat. F/17/1311 à $\left.\mathrm{F} / 17 / 1317^{2}\right)$.

${ }^{67}$ CRC, p. 44

${ }^{68}$ Sur la pratique jésuite, voir les mémoires d'Achile Dionis du Séjour, qui note pour chaque année passée au pensionnat de Louis-le-Grand entre 1743 et 1751, le nom de son professeur et de son préfet de chambre (Bibl. nat., ms lat. $\mathrm{n}^{\circ}$ 10992, fo 2 à 9). Pour les professeurs séculiers de collège, voir B. Noguès, op. cit., p. 59.

${ }^{69} \mathrm{C}$ 'est ce qui ressort du tableau dressé en 1763 (CRC). Voir aussi pour le cas parisien, B. Noguès, op. cit., p. 17.

${ }^{70}$ CRC, p. 32.
} 
de problèmes méthodologiques, qui n'ont pas tous pu être levés et qui constituent les limites de l'exercice. La valeur locative des bâtiments mis à disposition par la ville donne lieu à des estimations extrêmement variables et certainement très subjectives, qui reflètent peut-être la diversité des conditions matérielles de l'enseignement, mais font également intervenir la conjoncture immobilière, la ville d'implantation et les intentions de l'auteur de l'évaluation. Trop difficile à interpréter et à corriger de notre point de vue, cet aspect a donc été laissé de côté et les estimations qui suivent doivent s'entendre hors coûts mobiliers et immobiliers, en considérant que tous les collèges disposent d'une manière ou d'une autre de locaux pour assurer les classes. De même, la subvention indirecte de l'activité d'enseignement par le pensionnat reste obscure, car elle donne rarement lieu à des transferts financiers qui apparaîtraient dans les comptes - il s'agit bien souvent d'heures de travail volées au pensionnat - et parce que sa fréquence et son intensité sont impossibles à estimer. Afin de permettre des comparaisons acceptables, l'analyse a donc été réduite aux quatre sources de financement les plus communes présentées plus haut: le revenu de la préceptoriale et des bénéfices réunis aux collèges, c'est-à-dire ce qui vient de l'Église; l'écolage versé par les familles ; les fondations privées ; les subventions de la ville. L'étude a porté sur les recettes d'une trentaine d'établissements, saisis entre 1561 et 1792 , et a davantage l'ambition de fournir des ordres de grandeur que des estimations précises, tant est grande l'hétérogénéité de la documentation.

L'impression qui domine lors de la mise en série des recettes des établissements est l'extrême diversité des configurations, sans qu'aucun élément décisif comme la période, la taille de la ville, l'appartenance à telle ou telle congrégation ne suffise à départager les différents types de collèges. On retrouve ici l'extrême diversité des situations décrite à propos de l'école élémentaire ( «école en miette »). La manière la plus pertinente d'ordonner la série paraît la prise en compte du poids du financement municipal comparé à celui des familles (graphique 1). Un tel classement fait apparaître deux groupes de collèges assez homogènes, qui ne recoupe que partiellement le classement qu'avaient proposé M.-M. Compère et D. Julia, fondé il est vrai sur des critères différents (période de fondation, taille de la ville et fondateur principal $)^{71}$.

\footnotetext{
${ }^{71}$ M.-M. Compère, D. Julia, « Les collèges sous l'Ancien Régime », art. cit., p. 8, p. 15 et p. 23.
} 
Graphique 1

(la date correspond à l'année où le budget est analysé) ${ }^{72}$

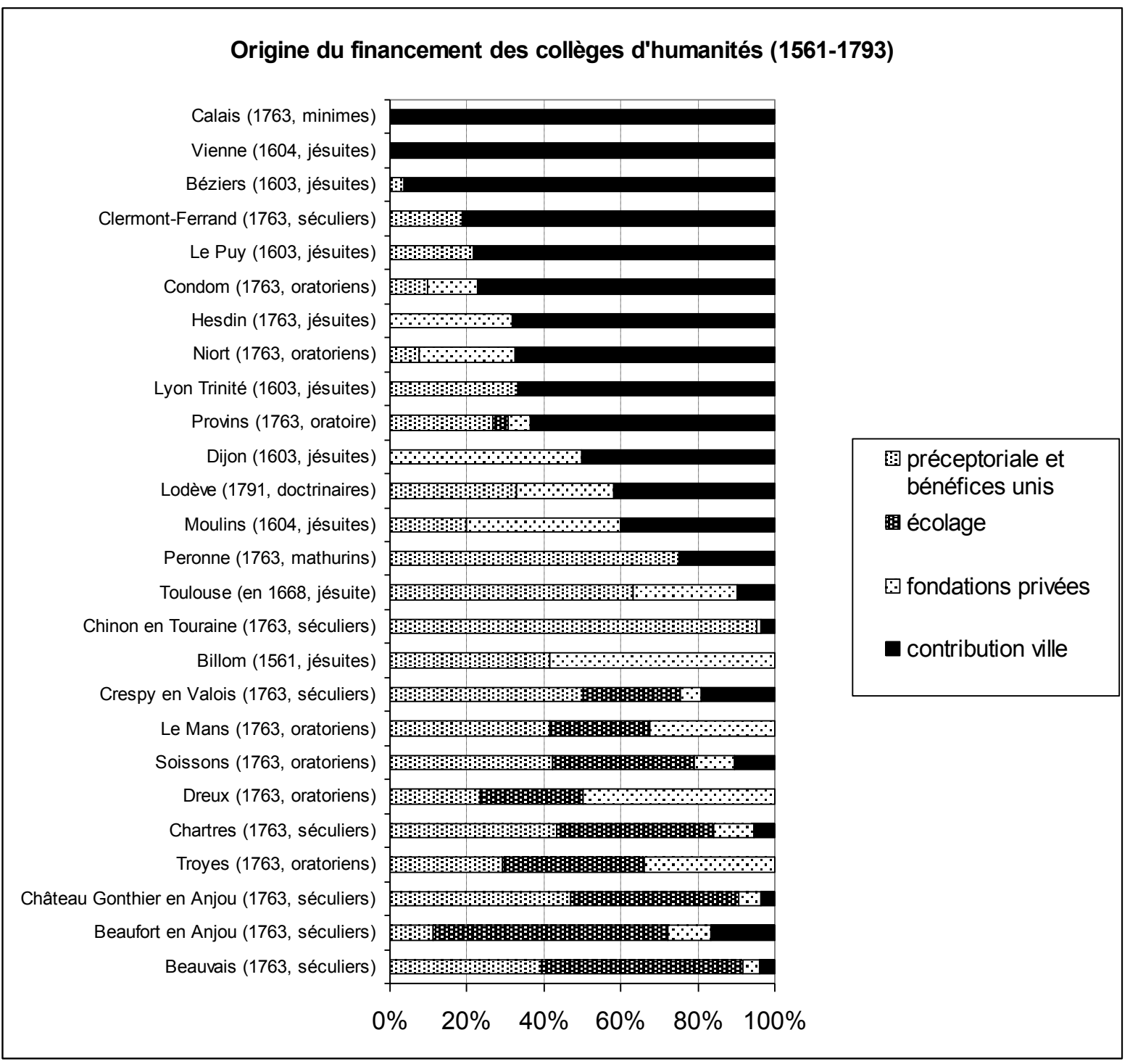

Le premier groupe (neuf collèges, de Beauvais à Crespy-en-Valois) est exclusivement composé d'établissements séculiers (cinq) ou oratoriens (quatre). Ils se caractérisent tous par la grande diversité des sources de financement, signe qu'aucune n'est suffisante pour assurer seule le fonctionnement de l'établissement, et signe également d'un empilement des revenus opéré au fil d'une histoire longue et complexe. On y relève la présence systématique et massive de l'écolage ( $41 \%$ des ressources à Chartres, $53 \%$ à Beauvais) et le poids des bénéfices réunis. En revanche, la faiblesse voire l'absence de subvention municipale est frappante : seulement $6 \%$ à Chartres, $4 \%$ à Beauvais, rien du tout à Troyes. Ces établissements sont en réalité les héritiers de structures plus anciennes, remontant souvent au

\footnotetext{
${ }^{72}$ Cette analyse des financements, qui ne prétend fournir que des ordres de grandeur compte tenue des réserves formulées plus haut, s'appuie essentiellement sur les rapports aux Parlements de 1763 (CRC), recoupés et complétés par les informations fournies par M.-M. Compère et D. Julia, Les collèges français..., op. cit.; H. Fourqueroy, op. cit.; État des collèges de la Compagnie de Jésus en Aquitaine, 1603, publié par C. de Rochemonteix, op. cit., p. 230 et sq.
} 
Moyen Âge, même si l'Oratoire a pu à un moment donné prendre la relève dans quelques établissements en déshérence. Le cas le plus typique est celui de Chartres, où le collège fait figure de lointain descendant de la célèbre école cathédrale, fondée dès le début du $\mathrm{XI}^{\mathrm{e}}$ siècle par Fulbert de Chartres. Cependant, malgré un passé parfois prestigieux, les villes qui hébergent ces établissements paraissent leur témoigner un faible intérêt, en refusant d'abonder au cours de la période moderne des ressources devenues manifestement insuffisantes.

Le second ensemble (douze établissements, de Moulins à Calais) présente des caractéristiques inverses, avec un nombre important de collèges jésuites ou d'autres congrégations et un seul établissement séculier, l'absence systématique d'écolage et la très forte contribution publique. Moins diversifiés que dans le groupe précédent, ces revenus peuvent certes intégrer des bénéfices réunis (généralement récupérés lors de la reprise d'un collège existant) ou des fondations privées (legs de particuliers accumulés au fil du temps, qui ne cessent de gonfler et dont on a vu que les jésuites de Lyon avaient prévu l'éventualité dès la fondation du collège), mais le trait marquant est bien la place de premier plan qu'occupe toujours la subvention municipale. Ces établissements, souvent fondés ou refondés au tournant du XVI ${ }^{\mathrm{e}}$ et du XVII ${ }^{\mathrm{e}}$ siècle, quand s'épanouit le goût des élites pour les humanités classiques, sont indiscutablement nés du désir des notables urbains de disposer localement d'un collège. Ils ont été prêts à y mettre le prix, quitte à le financer entièrement. Restent enfin, de Billon à Péronne, quatre établissements où le poids de la contribution urbaine est nettement moins fort. Mais l'absence d'écolage et la forte présence des jésuites montrent qu'ils s'apparentent fondamentalement au modèle qui vient d'être décrit. La richesse des revenus ecclésiastiques, parfois lié à la volonté d'un évêque de la Réforme catholique d'attirer dans sa ville telle congrégation, ou à la générosité de quelque donateur privé dispense simplement la ville de manifester un engagement particulier à travers de gros efforts financiers.

Ces quelques observations, fondées sur l'analyse des finances d'une trentaine de cas, mériteraient évidemment d'être approfondies. La documentation, abondante et accessible, permettrait sans peine d'élargir l'échantillon et d'affiner cette catégorisation, même si on peut s'interroger sur son utilité. Telle qu'elle existe, cette grille d'analyse, purement financière, permet néanmoins de dégager trois enseignements. Malgré un effort de catégorisation, le trait principal est bien l'extrême diversité des situations possibles. Des oratoriens de Troyes aux jésuites de Vienne, des séculiers de Chartres à ceux de Clermont-Ferrand, toutes les configurations peuvent se rencontrer. En l'absence de puissance unificatrice active à l'échelle du Royaume, les singularités héritées d'une histoire déjà pluriséculaire restent fortes. Ceci limite évidemment toute explication qui ferait reposer sur un unique acteur ou facteur le développement de ces collèges d'humanités. Dans ce cadre, on relève, plus que l'importance de l'investissement urbain - qui est loin d'être généralisée-, la nouveauté de cet investissement, qui fait contraste avec la période médiévale. Malgré le recul des libertés urbaines dans la monarchie absolutiste et le malthusianisme éducatif ambiant, il s'agit bien ici de l'irruption d'un nouvel acteur du monde éducatif à ce niveau d'enseignement. Outre les questions de prestige - la présence d'un collège ne serait-elle pas un des marqueurs du statut urbain ?-, cet investissement traduit finalement l'appétence connue des notables pour la formation aux humanités, ainsi que leurs réticences, à partir du XVII ${ }^{\mathrm{e}}$ siècle, à envoyer leurs fils se former dans une lointaine université. À côté de ce mouvement, très visible car nouveau, la place qu'occupe l'Église reste importante, mais elle est devenue plus complexe : les représentations communes, comme sa contribution effective, à travers l'union de bénéfices, en font toujours un acteur majeur du financement des collèges. Mais les modalités de sa participation, qui résident désormais dans des transferts d'institution à institution, préparent la 
voie à la laïcisation des enseignants, observée dès le milieu du XVIII siècle. Enfin, quel que soit le poids de ces deux contributeurs «publics», une analyse des coûts globaux de l'éducation montre de toute façon qu'une part très importante des dépenses est, directement ou indirectement, supporté par les familles. Si cet investissement familial est patent dans l'entretien matériel des enfants, de moins en moins subventionné par les bourses, il se manifeste également par le financement des cours d'agrément, ou à travers le transfert des ressources du pensionnat vers l'exercice. On estime que dans la France du début du XXI siècle l'État apporte $60 \%$ du financement de l'éducation, les collectivités territoriales $20 \%$, les ménages $8 \%{ }^{73}$. Établir une telle estimation pour la période moderne serait extrêmement périlleux. Comment tracer le périmètre de l'enseignement proprement dit, de l'éducation en général, de l'entretien des enfants, compte tenu de la porosité de ces différents postes ? Où placer la pension ou les cours d'agrément, dont le caractère indispensable dépend du milieu social retenu? De plus, une moyenne ou une estimation globale auraient-t-elles un sens, au vu de l'hétérogénéité des situations repérées? On ne s'y risquera pas, conscient qu'après avoir analysé les comptes de quelques centaines de collèges, on obtiendrait certainement une moyenne statistiquement fondée, mais qui ne dirait rien des pratiques des établissements ou des familles, et serait donc dénuée de sens.

\section{Boris NOGUÈS}

\footnotetext{
${ }^{73}$ Roger-François Gauthier, André Robert, L'école et l'argent. Quels financements pour quelles finalités ?, Paris, 2005, p. 107.
} 